\title{
MedienPädagogik
}

Zeitschrift für Theorie und Praxis der Medienbildung www.medienpaed.com

\section{Informatische Modellbildung als Dimension einer künstlerisch-technisch konzipierten Medienbildung}

Daniela Reimann

\begin{abstract}
Zusammenfassung
Heute sind nicht nur diverse gestaltungsbezogene Disziplinen gefragt, sich aktiv mit der Digitalisierung von Information und ihren Potentialen und Nebenwirkungen für Bildungsprozesse auseinander zu setzen. In Deutschland haben sich Technikdidaktik und informatische Bildung getrennt von Medienpädagogik und Mediendidaktik entwickelt und wurden entsprechend unabhängig voneinander betrieben. Dies gilt auch für die Ästhetische Bildung, die informatisch-technische Inhalte kaum je curricular in die Auseinandersetzung mit digitaler Medienkultur integriert hat, schon gar nicht systematisch. Im diesem Artikel wird, ausgehend vom Stand der Forschung (zur Integration informatischer Inhalte in die Medienbildung) und den identifizierten Grundproblemen der Medienbildung zwecks Kompensation der Defizite, ein auf Gestaltung basierter Ansatz präsentiert, kontextualisiert und diskutiert, der Technikverständnis in ein Konzept integrierter Medienbildung einbezieht, dies als Bildungsziel im Sinne eines ganzheitlichen Verständnisses von Welt begreift und daher disziplinüberschreitend angelegt ist. Der Ansatz geht über technokratische Vorstellungen einer Technikdidaktik hinaus, die Medientechnologien isoliert von lebensbedeutsamen, ästhetischkünstlerisch gestalteten Kontexten betrachtet und vermittelt. Im didaktischen Konzept werden die Grundlagen informatischer Modellbildung als relevante Teilbereiche einer zeitgemässen, am Subjekt orientierten, gleichermassen ästhetisch-künstlerisch und technisch-informatisch geprägten Medienbildung aufgewiesen, ihre pädagogischen Vorläufer, Forschungsprojekte und Nachbardisziplinen benannt sowie Ausblicke für eine zeitgemässe Medienbildung gegeben.
\end{abstract}

\section{Computational modelling as a dimension of an integrated art and computer science based media education}

\begin{abstract}
Today, not only diverse design-related disciplines are required to actively deal with the digitization of information and its potentials and side effects for education processes. In Germany, technology didactics and computer science education developed separately from media education and were taught isolated from each other. This also
\end{abstract}


applies to the art education, wherein computer science based learning contents to examine the digital media culture, hardly ever got integrated into the curricula. Based on the current state of research, in this article, a design based approach is presented, contextualized and discussed, which perceives the understanding of technology in connection with an integrated media education, in terms of a holistic understanding of the world, therefore applied across disciplines. It steps beyond a technocratic view of computer education, which excludes meaningful contexts of consideration and communication. The didactic approach, the basics of computer science modelling will be recognized as relevant parts of a contemporary, subject-oriented, both art and technology-based media education. Educational precursors and related disciplines are named, and in conclusion an outlook at contemporary media education is given.

\section{Einleitung}

Die Digitalisierung der Welt lässt erweiterte Kommunikations- und Handlungsräume entstehen, die zunehmend komplexer werden und damit für die interagierenden Subjekte schwierig zu erfassen und zu begreifen sind. "Software takes command» postulierte Lev Manovich bereits 2008'. Inzwischen hat sich die digitale Technik geradezu in unsere Existenzen eingeschrieben, sie hat alle gesellschaftlichen und privaten Räume wie Arbeit, Freizeit, Medizin, Gesundheit und Bildung besetzt und die mediale Durchdringung aller Lebensbereiche (Mediatisierung) hat auch die Kultur selbst technologisch werden lassen (vgl. Richard/Bruns 2004, 71). Es sind nicht nur die wissenschaftlichen Disziplinen gefragt, sich aktiv mit der Digitalisierung von Information und ihren gewünschten Effekten und unerwünschten Nebenwirkungen auseinander zu setzen. Das allgemeine Technikverständnis zu fördern, um eine gestaltende Teilnahme an diesem gesellschaftlichen Prozess zu erreichen, liegt nicht nur als Aufgabe für die Medienpädagogik nahe. Programmierkompetenz, auch «code literacy», gilt inzwischen nicht nur unter Informatikern als Schlüssel zur Befähigung von Partizipation im Sinne der Technikgestaltung. Sie beinhaltet die Fähigkeit, Computer per Programmierung selbst zu steuern und soll das Verständnis dafür, wie Computer und Softwareprogramme funktionieren, fördern. Das mit Programmierkompetenz verknüpfte Bildungsziel bezeichnet weniger ein technokratisches Verständnis, wie etwa das Beherrschen diverser Programmiersprachen, sondern zielt vielmehr auf eine damit verbundene Metakompetenz - die Förderung von "Computational thinking», die Bildung von Modellen und das Anwenden von logischen Strukturen ab. Aus diesem Grund verfolgen Projekte wie "TACCLE3 coding» die Vermittlung der genannten Ziele an Grundschullehrkräfte, um Programmierkompetenz bei Multiplikatoren/-innen zu fördern und

1 In gleichnamigem Buch analysiert er den Bereich der «Software Studies» und thematisiert die kulturelle Prägung der uns umgebenden Computerprogramme als «Cultural Software». 
Technikinteresse bereits im Kindesalter wecken zu können (García-Peñalvo 2016, Vermeersch/Hughes 2015).

Die Diskussion um Programmierfähigkeit wird inzwischen auch in Deutschland geführt, um gesellschaftliche Teilhabe und Mitgestaltung zukünftiger Kommunikations- und Handlungsräume durch die heranwachsende Generation zu verbessern. Die den digitalen Technologien zugrundeliegenden Algorithmen werden teilweise sogar als "Grundlage einer neuen Weltsprache verstanden» (vgl. Kreye 2014). Das war nicht immer so. Programmierfähigkeit wurde zwar einerseits bereits als 4 . Kulturtechnik - neben Lesen, Schreiben und Rechnen - bezeichnet und dies auch im Kontext der Abhängigkeit technischer Entwicklungen von gesellschaftlichen Anforderungen analysiert (vgl. Coy 2008) oder in Frage gestellt. Andererseits hat bisher keine systematische Integration von Programmierkompetenz in allgemeinbildende schulische Curricula in der Sekundarstufe 1 oder in der Grundschule in Deutschland stattgefunden. Auch ohne den Erwerb von Programmierfähigkeit mit Lese- und Schreibkompetenz in seiner Bedeutsamkeit gleichzustellen, lässt sich das mit ihr verbundene Potenzial für kreative Prozesse und Teilhabe schwer von der Hand weisen. Es ist eben ein Unterschied, ob jemand ein Gerät bedienen oder aktiv steuern und für bestimmte Zwecke manipulieren kann.

\section{Programmierfähigkeit als Aspekt der Medienbildung}

Programmieren als Lerninhalt hat gerade bei weniger technikaffinen pädagogischen Zielgruppen unterschiedlicher Altersstufen und Geschlechts einen zweifelhaften bzw. negativ konnotierten Ruf. Das Image ist noch immer mit den Vorstellungen und Klischees aus den Anfängen der Computerkultur behaftet. Turkle konstatierte bereits 1984, das die algorithmische Maschine eine neue Computerkultur begründet und die Gesellschaft in Technikfreaks, Kritiker und Ahnungslose spalte. Einerseits hat die Maschine unser Leben stark verändert, wir tauchen in simulierte Welten ein und interagieren mit zunehmend vernetzten Artefakten in Beruf und Freizeit. Andererseits war das Wissen über die Funktionalität digitaler Technologie den Informatik-Experten vorbehalten. Turkle betonte in Bezug auf die Rezeption der Computertechnologie, unter Rückbezug auf die Geschichte der Medien, dass der Computer zum neuen kulturellen Symbol der bedrohlichen Wirkungen geworden ist, die Rousseau dem Schreibstift zuschrieb: Verlust des unmittelbaren Kontaktes zu anderen Menschen, Erschaffung einer eigenen Welt, Abkehr von den realen Gegenständen und Hinwendung zu ihrer symbolischen Repräsentation. Mit dem Programmieren, wie mit so vielen anderen Aspekten, greift die Präsenz des Computers Dinge auf, die bereits vor seinem Erscheinen Gegenstand der Besorgnis waren und verleiht ihnen eine neue Gestalt und ein neues Ausmass. Wenn unsere Vorstellungen von Kindheit durch schreibende Kinder in Frage gestellt 
werden, was bewirken dann programmierende Kinder? Wenn die Unschuld der Kindheit schon durch das Schreiben angegriffen wird, um wieviel mehr nimmt sie dann durch das Programmieren schaden? (Turkle 1984, 115).

Turkle stellte eine universelle Wirkung von Computern auf Kinder in Frage und ging subjektorientiert der Frage nach, was verschiedene Typen von Kindern mit Computern machen. Sie beobachtete vor allem den Umgang der Kinder mit der von Papert entwickelten Programmiersprache LOGO, die es dem Kind ermöglichte, dem Computer etwas «beizubringen». In der Austen-Schule realisierte Turkle das Entstehen einer intellektuellen Praxisgemeinschaft, "wie sie unter Schulkindern normalerweise nicht zu finden ist» (Turkle 1984, 122) und wies darauf hin, dass «am bemerkenswertesten an dieser Gemeinschaft die Tatsache [ist], daß sie Kinder umfaßt, die in ihrer Persönlichkeit, ihren Interessen, ihrer Art zu lernen sehr große Unterschiede aufweisen, Unterscheide, die deutlich in ihren Programmierstilen zum Ausdruck kommen» (Turkle 1984, 123). Unterschiedliche Programmierstile von Kindern wurden als ein Ausdruck von Persönlichkeit begriffen, zu einer Zeit, als in Deutschland in Erziehungswissenschaft und Medienpädagogik eher Film, Fernsehen und später Video im Hinblick auf Analyse, Kritik und in der Medienpraxis Video-Produktionen thematisiert wurden.

Die Thematisierung informatischer Modellbildung als ein relevanter Lernbereich im Kontext zeitgemässer Medienbildung, die auch ganz aktuelle technologische Entwicklungen integriert, wurde in der Medienpädagogik bis 2001 zunächst nicht aufgegriffen. Digitale Medien - als «Neue Medien» betitelt - wurden und werden an Hochschulen im Bereich der Studiengebiete curricular weitgehend mit den analogen Medien vermischt, so dass die Auseinandersetzung mit ihren medienspezifischen Charakteristika - die Programmiertheit und Programmierbarkeit, die sie mit Interaktivität, also Verhalten ausstattet - nicht nur mediendidaktisch in den Hintergrund gerieten.

Eine positive Bewertung von Programmierfähigkeit war dagegen auf internationaler Bühne besonders in der, von der konstruktivistischen Pädagogik geprägten, konstruktionistischen Technikdidaktik festzustellen, die vor allem durch die Arbeiten von Papert (1982), auf seinen Beiträgen zur Lernforschung und Programmierkompetenz von Kindern basiert, die er in Auseinandersetzung mit den Lerntheorien Piagets entwickelt und bereits in den 1980er Jahren in Schulen sozialer Brennpunkte in den USA erprobt hat. Dort entwickelte sich in seiner Nachfolge, insbesondere am MIT MediaLab, eine Kultur der kreativen Technikvermittlung, bei der gerade spielerische und gestalterische Kontexte integriert wurden. Beispielhaft in Nachfolge der programmierbaren «turtles» von Papert entstanden «programmable LEGO bricks» (Resnick et al. 2000, 13), programmierbare Bausteine, die später von $\angle E G O^{\circledR}$ als Mindstorms-Gruppe kommerzialisiert wurden. Sie fanden in den letzten 10 Jahren auch an Schulen in Europa zunehmend Verbreitung. Auch 
Scratch, ein Programm, das Programmierung von Animationen mit dem Erzählen von Geschichten (Storytelling) verbindet, wurde von der MIT-Forschungsgruppe «Lifelong Kindergarten» entwickelt und wird im Rahmen einer zu einer grossen Anwender-Community weiterentwickelt und für Schulen inkl. didaktischem Material bereitgehalten. In den USA hat sich das "Coding» als Lernziel vor allem im Rahmen der «Learning Sciences» und Lernforschung etabliert. Die «Lifelong Kindergarten»Gruppe hat sich die Bewahrung und explizite Integration spielerischer Elemente und kindlicher Spielzeuge zum Forschungsbereich erhoben: Resnick u.a. haben sich in der Tradition Paperts zum Ziel gesetzt, die Grundlagen für die Förderung von Kreativität zu legen und entsprechende digitale Werkzeuge für kreative Prozesse zu entwickeln, bei denen das eigenständige Programmieren durch die Lernenden selbst im Mittelpunkt steht: (siehe MIT-Website des LLK 2015)².

Programmierkompetenz als Lernziel wurde von Papert bereits in den 1980er Jahren als Fähigkeit zur freien Steuerung und Manipulation des Computers durch das Kind selbst beschrieben, in einer Zeit, in der Kinder im Unterricht vorgefertigte Lern- bzw. Drill-Systeme durchlaufen sollten. Nicht der Computer sollte nach Papert das Kind steuern, sondern das Kind den Computer und damit Gestaltungsmöglichkeiten erhalten. Durch Paperts Arbeiten und seine zahlreichen Nachfolger/innen besteht in den USA bereits eine längere Tradition der Verbindung von Programmierfähigkeit und kreativen Prozessen. Ausserdem gibt es Initiativen zur Integration von Programmierkompetenz ins Curriculum an Hochschulen, wie z. B. in der Elektrotechnik am MIT, Forschungsgruppe High-Low-Tech (vgl. Buechley et al. 2008). Die Tradition der Wertschätzung informatisch-technischen Denkens und Handelns jenseits einer technokratischen Betrachtung wurde mittels kindgerechter Benutzeroberflächen und Mensch-Maschine-Schnittstellen umgesetzt, aber in Deutschland ausserhalb der Technikdidaktik kaum rezipiert. In "connected code» betonen Kafai und Burke (2014), das der Prozess des Programmierens, früher als ein obskures Handwerk, einsamer Technik-Freaks (Nerds) betrachtet, heute von Pädagogen/-innen und Theoretiker/innen nicht nur als eine entscheidende Fähigkeit für das Aufwachsen in der digitalen Medienkultur anerkannt ist, sondern auch als eine neue Form der Literalität, im Sinne einer Lese- und Schreibfähigkeit, als eine Art Alphabetisierung für das digitale Medium erkannt wurde, die als Lernziel für alle Kinder betrachtet wird. Programmierung wurde an K-12-Schulen in den USA bisher als eine Möglichkeit verstanden, das so genannte «informatische Denken» zu fördern, welches inzwischen zum Oberbegriff für das generelle Verständnis von Informatik und ihrer Bedeutung in einer sich ständig wandelnden, zunehmend digitalen Welt, geworden ist. Kafai und Burke (2014) plädieren über die blosse Befähigung zur Programmierung («coding competence») hinausgehend für das

2 URL: https://www.media.mit.edu/research/groups/lifelong-kindergarten [10.08.2015]) 
Programmieren-Können im Kontext des Netzes und der Partizipation («coding participation»), das der Realität und Lebenswirklichkeit der Jugendlichen heutzutage angemessener sei. Programmierung bewegt sich demnach im Kontext von Partizipation über das einzelne Subjekt hinaus, auf Ebene sozialer Netzwerke und die «Do-it-Yourself-» und «Maker-Kultur» der digitalen Welt. Es bedeutet für die Jugendlichen damit Teilhabe als aktiv Gestaltende.

\section{Informatische Modellbildung als Bildungsziel - die Debatte in Deutschland}

Trotz des festgestellten Fachkräftemangels gab es bisher nur begrenzt Konsequenzen für das deutsche Bildungssystem. In Deutschland verkündete Hamburgs Schulsenator Ties Rabe 2014, dass «Informatik in Hamburger Stadtteilschulen kein Pflichtfach mehr sein soll». Dem wurden Reaktionen wie «eine weitere Fremdsprache ist wichtiger als eine Programmiersprache» entgegengesetzt und Behördensprecher Peter Albrecht liess verlauten, Informatik sei «nicht für alle in gleicher Intensität vonnöten. Salopp formuliert: In einer mobilen Gesellschaft solle jeder Auto fahren können, aber nicht jeder müsse wissen, wie ein Wagen funktioniert» (vgl. Dahlmann 2014). An vielen Schulen würde ausschliesslich der «Umgang mit Microsoft-Office-Produkten und ein bisschen Google gelehrt» (a. a. O.). Bundesländer wie Bremen, Rheinland-Pfalz, Thüringen und Nordrhein-Westfalen verfolgen einen integrativen Ansatz. Computerkenntnisse, so die Forderung, sollten in anderen Fächern mitvermittelt, also nebenbei thematisiert werden. In Hessen gibt es ein Wahlangebot Informatik nur für die Klassen 5 bis 10 an Gymnasien. Und im Bildungsplan von Baden-Württemberg steht geschrieben, dass «Schüler sich privat schon ausreichend qualifizierten» (a. a. O.). Einige Bundesländer wie Bayern sind weiter fortgeschritten, andere nicht. Resümierend lässt sich feststellen, dass das Schulfach Informatik in Deutschland keinen hohen Stellenwert hat. Von Seiten der Deutschen Gesellschaft für Informatik gab es nicht erst 2007 Bemühungen, wurde die schulische Informatikdidaktik zu fördern.

2015 gab die grosse Koalition nach Rezeption der Ergebnisse der «International Computer and Information Literacy Study (ICILS)», die deutschen Schülerinnen und Schülern Kompetenzen beim Umgang mit dem Computer unterhalb des EUDurchschnitts nachwies, bekannt, dass im Rahmen des «Pakts für digitale Bildung» die «Förderung eines zeitgemäßen und altersgerechten Informatikunterrichts ab der Grundschule» realisiert werden soll, um «das Verständnis der Informatik und der Logik von Algorithmen als der Sprache der digitalen Welt» für einen kompetenten und «selbstbestimmten Umgang mit der Digitalisierung» zu fördern sei. Bisher spielten «der Umgang mit dem Internet und die Arbeit mit digitalen Lernmaterialien im Unterricht oft noch eine eher untergeordnete Rolle», so lautet es im Antrag (Roßmann 2015). Deutschland habe einen grossen Nachholbedarf, da 
digitale Bildung «noch viel zu sehr als Orchideenthema betrachtet würde» (ebenda). Relevant in der Diskussion ist dabei aber über die geforderte Ausstattung (z. B. Breitbandanschluss für alle Schulen ${ }^{3}$ ) hinaus vor allem die didaktische Ebene und was Schülerinnen und Schüler mit bestimmten Computertechnologien im Unterricht zu Bildungszwecken tun.

\section{Theorie und Praxis integrierter ästhetischer und informatischer Aus- und Fortbildung}

Im Rahmen des BLK-Modellversuch «Theorie und Praxis integrierter ästhetischer und informatischer Aus- und Fortbildung (ArtCom)» ${ }^{4}$ im Jahr 2001 wurde informatische Modellbildung erstmals in Deutschland als Lerninhalt und relevanter Teilbereich einer ganzheitlichen Medienbildung, die auf einem tiefgründigen Technikverständnis beruht, thematisiert und an allgemein bildenden Schulen im Fach Kunst spielerisch und mit ästhetischen Verfahren und stärker künstlerischen Kontexten verknüpft vermittelt (vgl. Reimann 2006, Reimann et. al. 2003, 2004). Das didaktische Konzept des Modellversuchs basierte auf der Einsicht, einen andersartigen Umgang mit Computertechnologien jenseits der auf blosse Anwenderkompetenzen abzielenden schulischen Praxis zu konzipieren, zu erproben und zu evaluieren. Dazu wurde eine disziplinübergreifende Kooperation realisiert, so dass eine gleichsam ästhetisch und informatisch geprägte Medienbildung umgesetzt werden konnte. Der Computerunterricht wurde dabei bewusst aus den Computerfachräumen herausgeholt und nicht, wie sonst üblich, isoliert betrachtet und vermittelt. Vielmehr wurde die abstrakte semiotische und algorithmische Maschine als ein gestaltbares, programmierbares Medium im Unterricht thematisiert und erprobt. Es wurden die Disziplinen Kunst, Gestaltung und Informatik im Schulfach Kunst an allgemeinbildenden Schulen in Schleswig-Holstein zusammengeführt und fächerübergreifend mit Lehrkräften im Rahmen unterschiedlicher pädagogischer Settings erprobt und evaluiert. Die integrierte ästhetisch-informatische Medienbildung mit der Verknüpfung zweier zunächst ungleich erscheinenden Arbeitsverfahren wurde eingeführt: Das freie Arbeiten in ästhetisch-künstlerischen Entwurfsprozessen und die präzise Berechenbarkeit informatischer Modellierung,

3 Die Forderung erinnert an die in den frühen 2000 er Jahren lancierte «Ausstattungsinitiative» «Schulen ans Netz», die zunächst auf die Implementierung einer grundlegenden IT-Infrastruktur an Schulen abzielte, ohne gleichzeitig didaktische Konzepte oder Konsequenzen für die Lernkultur mit zu entwickeln.

4 Die BLK-Initiative "Kulturelle Bildung im Medienzeitalter» zielte auf eine systematische Einbeziehung der Künste und Ästhetisch-Kulturellen Bildung im Kontext. Bundesweit wurden in den Ländern Modellversuche zur Entwicklung, Erprobung und Evaluation neuer Bildungsmassnahmen und -szenarien aufgelegt und gefördert. Im zugehörigen Gutachten zum Programm betont Pazzini, dass die «Kulturelle Bildung Möglichkeiten und Ressourcen [beinhaltet], die in den Wissenschaften, insbesondere den Naturwissenschaften, der Mathematik und den technisch ausgerichteten Wissenschaften nicht oder nur untergründig zur Verfügung stehen.» (Pazzini 1999, 5) 
wobei keines der Verfahren dem anderen über- oder untergeordnet wird. Die zeitgenössische Medienkunst als disziplinübergreifendes Genre diente dabei als Impulsgeber und wird didaktisch für eine Medienbildung herangezogen, die jenseits von Fächergrenzen verortet ist und dabei gerade Sinn und Sinnlichkeit von Einsatzweisen, Materialien und «Multi-Medien» einbezieht (vgl. Reimann 2006). Über das blosse Verwenden vorgefertigter medialer Applikationen und Artefakte hinausgehend, wurde die Mediengestaltungspraxis auf die Ebene der Programmierung ausweitet. Gleichzeitig wurden ästhetische Prozesse und künstlerische Kontexte an Schulen als Zugänge zur Informatik angeboten. Programmieren bedeutet, den digitalen Objekten und Artefakten Verhalten zuzuweisen. Interaktivität als die Haupteigenschaft des digitalen Mediums zu vermitteln. Digitale Technologien, neue Schnittstellen und geeignete Interfaces wurden selektiert, die in der Schule in Deutschland bis dahin nicht nur in den Fächern Kunst und Informatik nicht eingesetzt wurden. Eingesetzt wurden aktuelle Medientechnologien, die die eigenständige Konzeption, Gestaltung, Konstruktion und Programmierung von medialen Objekten mit der Verbindung physischer und digitaler Räume durch die pädagogischen Zielgruppen unterstützen. Die angebotenen digitalen Technologien waren Robotik, Hypermedia-Systeme wie 3D-Internet-Welten sowie interaktive Identitäten sowie später mobile Medien. Technologien wurden dabei als explorative Räume für ästhetische Erfahrungen angeboten und vermittelt. Leitmotive für die Wahl der Digitalen Technologien waren:

- die eigenständige Programmierbarkeit mittels ikonischer Programmierumgebungen und Interfaces (Visualisierung von informatischer Modellbildung),

- Low cost und Open-Source-Software-Lösungen,

- Systemimmanente Unterstützung der Verknüpfung mit real-physischen Räumen,

- Gestaltbarkeit: Es wurden keine vorgefertigten Applikationen eingesetzt, sondern fantasievolle Eigenkreationen von Medienobjekten von den Schülerinnen und Schülern erfunden und im Rahmen von interaktiven Installationen, Environments, Lernräume als Mixed Reality-Erlebnisräume realisiert.

Interdisziplinäre künstlerische Forschungsanordnungen wurden initiiert und experimentell zusammen mit den Lehrkräften umgesetzt. So genannte Mixed Reality-Lernräume (Reimann 2006, Reimann et al. 2003) wurden auf Basis von Lowcost-Modellen und selbst konstruierten Umgebungen in Schulen realisiert und erforscht. Ausgehend von der gemeinsamen Schnittfläche von Kunst und Informatik - der Semiotik und des Zeichenbegriffs (vgl. Amelunxen/Herczeg 2001) basierte der Modellversuch an Schulen auf der Kooperation dreier Hochschulen ${ }^{5}$,

5 Das Institut für Multimediale und Interaktive Systeme IMIS der Universität zu Lübeck, die MuthesiusHochschule für Kunst und Gestaltung (Kunstlehrerausbildung an Gymnasien) sowie die ChristianAlbrechts-Universität zu Kiel (Kunstlehrerausbildung an Gymnasien und Realschulen). 
so dass die als Schlüsseldisziplinen der zunehmenden Digitalisierung und Gestaltung von Information identifizierten Studiengebiete zusammengeführt und mit der Lehrerausbildung verbunden wurden. Erstmalig in Deutschland sollte im Rahmen der Medienbildung bei Kindern und Jugendlichen ab dem Grundschulalter ein grundlegendes Verständnis von Technik und informatischen Modellen durch das eigenständige Gestalten, Konstruieren und Programmieren der algorithmischen Maschine erlebt und im Kunstunterricht als Teil ästhetisch-künstlerischen Handelns mit bildnerischen Mitteln vermittelt wurden. Anders als im herkömmlichen Technik- und Informatikunterricht wurde Programmierung hier nicht durch das übliche Erlernen komplexer (textbasierter) Programmiersprachen verstanden, sondern durch visuelle Formen der Repräsentation und einfacher Handhabung mittels Drag-and-Drop-Verfahren ersetzt: Ikonische Programmierung, bereits von Canfield Smith (1975) im Kontext kreativer Programmierumgebungen (Pygmalion) reflektiert, wurde aufgegriffen und als eine Voraussetzung für die Selektion schüler/innengerechter, geeigneter Software herangezogen (vgl. auch Reimann 2006, 126f.), die die Transparenz der Blackbox Computer und die Visualisierung informatischer Modelle unterstützten. Mit Beginn des Modellversuchs ArtDeCom ging die Verwendung des Computers gerade über ihren Einsatz als blosses Werkzeug, Ressource oder Speichermedium hinaus. Medien zu programmieren heisst, ihnen Handlungsanweisungen zu geben und Prozesse des kontinuierlichen Überprüfens und Überarbeitens von Software (Debugging) auszuführen. Erstmals wurden im Modellversuch auch neuartige, z. B. haptische und auditive Schnittstellen in die Medienbildung integriert und «Mixed Reality-Konzepte» für digital erweiterte Lernräume (Reimann 2006) eingesetzt, so dass der Körper und seine performativen Prozesse sowie die physische Lernumgebung stärker einbezogen werden konnten als das am herkömmlichen Einzelarbeitsplatz vor dem Bildschirm möglich war ${ }^{6}-$ zu einer Zeit vor der Kommerzialisierung neuer Interaktionsformen ${ }^{7}$. Heute spiegeln sich disziplinäre Vermischungen von ästhetisch-künstlerischem und informatischtechnischem Denken und Handeln u. a. in den aktuellen Trends und medialen Praxen und Praxisgemeinschaften, z.B. im Bereich der «Maker-Kultur» (siehe Schön et al. 2016), die auf dem Erfinder-Gedanken basiert (Anderson 2013), in Form der so genannten FABRICATION (FAB)-LABS und «Do-it-yourself» (DIY)-Initiativen, im Rahmen neuer Bildungsinstitutionen (z.B. Hacker-Akademie Hamburg) sowie in neuen Studiengebieten ${ }^{8}$. In die Schule haben diese Praxen der Mediengestaltung

6 Z.B. über Touch- und Geräuschsensoren im Kontext interaktiver Systeme und Tangible Media-Konzepte.

7 Wie z.B. der Wiimote, der Gamecontroller (Wii-Remote) für die 2006 veröffentlichte Spielkonsole Wii von Nintendo (vgl. Reimann 2011) sowie Kinect (von dt. Kinetische Verbindung) zur Steuerung der Videospielkonsole Xbox360, die 2010 auf den Markt kam.

8 Z.B. Smart Textile als Anwendungsbereich der Elektrotechnik und die verwendete Arduino-Technologie als Programmierumgebung in kreativen Prozessen: http://web.media.mit.edu/ leah/publications/buechley_ITiCSE_07.pdf. 
bisher kaum systematisch Eingang gefunden, sie werden vielmehr im Rahmen von Forschungsprojekten, ausserschulischen kommunalen oder städtischen Laboratorien (z. B. fabrication/Fab Labs) realisiert.

\section{Grundprobleme der Medienbildung}

In den Schulen stellt die kontinuierliche Einbindung kreativer Auseinandersetzungen mit Technik wie «Maker-Aktivitäten» und das experimentelle Arbeiten mit Technologien wie Arduino im Kontext von kreativen Prozessen (Reimann/Maday 2016) oder Gestaltungen im Technikunterricht (Przybylla/Romeike 2013) weiterhin eher die Ausnahme dar. Es lassen sich grundlegende Herausforderungen bei der Integration von Medien in Lehr-Lernprozesse (Mediendidaktik) und der Untersuchung und Erprobung der Medien selbst als Unterrichtsgegenstand im Rahmen von Mediengestaltung und Medienforschung beobachten. Der schnelle technische Wandel mit immer grösseren Speicherkapazitäten sowie die Digitalisierung der physischen Welt zog neue mediale Praxen und Trends nach sich und sorgte in unterschiedlichen Disziplinen für eine verstärkte Auseinandersetzung mit dem Thema Computertechnologie in der Bildung. Wurde zeitweise noch die Einführung eines eigenständigen Unterrichtsfachs Medien gefordert und die Initiative «Keine Bildung ohne Medien» vorangetrieben (Niesyto/Moser 2009), so wurde andererseits festgestellt, dass sich alle Disziplinen mit dem Computer und seinen Wirkungen auseinandersetzen müssten: Medienbildung als Querschnittskompetenz wäre demnach horizontal in Lehr-Lernprozessen zu verorten und nicht isoliert als Einzelfach zu unterrichten. Die Informatik-Didaktik (Lehrerausbildung) wurde an Universitäten zeitweise stiefmütterlich behandelt. Seit einigen Jahren gibt es von Seiten der Informatik zunehmend Initiativen, die Integration der informatischen Bildung in die allgemeine schulische Bildung der Bundesländer anzuregen, um Lehrende und Lernende zu fördern (z. B. durch den Fachausschuss Informatische Bildung und Schulen (IBS) der Deutschen Gesellschaft für Informatik). Es handelt sich dabei eher um Aktivitäten zu schulinformatischen Kernthemen, die darauf abzielen den Dialog zwischen Wissenschaft und Schule anregen. Der Ausschuss hat aber vor allem auch Standards für die Informatik in der Schule definiert (IBS auf der Website der Gl) ${ }^{9}$. Die Einführung des Computers in der Schule wurde ab dem Jahr 2000 von Initiativen wie «Schulen ans Netz» bestimmt, so dass vor allem Geräte und Infrastruktur in Schulen bereitstanden. Damals standen den neuen Geräten (vorwiegend multimediafähige Personal Computer) noch keine mediendidaktischen Konzepte gegenüber, geschweige denn die Idee, Medientechnologie über den Einsatz von Computern im Unterricht hinausgehend selbst zum Gegenstand des Unterrichts zu machen. Grundprobleme des Computereinsatzes und damit

9 http://informatikstandards.de/index.htm?section=standards\&page_id=3 [2.10.2016]. 
der Medienbildung in Lehr-Lernkontexten wurden sichtbar. Der Computer im Unterricht wurde ausschliesslich als "computerunterstützter Unterricht (CUU)» gedacht, zu verstehen als «programmierte Unterweisung und Übungsprogramme» (von Alemann/Schatz 1986, 380), wie auch «computergestützte Lernprogramme». Im Folgenden werden wesentliche Grundprobleme der Medienbildung identifiziert und dargestellt.

\section{Einseitige Verwendung der Computertechnologie in Lehr-Lernprozessen}

Nicht nur in der Medienpädagogik und Ästhetischen Bildung wurde die Computertechnologie zunächst vornehmlich unter dem Aspekt des Einsatzes «Neuer Medien» in Bildungsprozessen reflektiert. Diese Einschränkung auf den Aspekt der Mediennutzung ist verbreitet und zieht auch die Untersuchung des Nutzerverhaltens unterschiedlicher Zielgruppen nach $\operatorname{sich}^{10}$. Darüberhinausgehend wurde der Computer mediendidaktisch kaum je über die Verwendung als ein blosses Werkzeug (z. B. als Schreibgerät, Werkzeug für die Bild- und Filmbearbeitung) oder als Ressource (Informationsträger, Archiv und Speichermedium) hinausgehend als ein digitales, programmiertes, mit Verhaltenden ausgestattetes Medium an Lernende vermittelt: "An vielen Schulen wird nur noch der Umgang mit Microsoft-OfficeProdukten und ein bisschen Google gelehrt» (Dahlmann 2014). Das Hilfsmittelverständnis, das die Nutzung von Computertechnologie als blosse Unterstützung von Lernprozessen betrachtet, ist in dem Begriff des «computergestützten Unterrichts» und der «mediengestützten Lernangebote» (z. B. Kerres 2012) ebenso auf den Punkt gebracht, wie im Anglizismus «E-Learning», als Darreichungsform von Lehr-Lernangeboten. Das pädagogisch geprägte Nachdenken über Medien in Lernprozessen war vom Hilfsmittelbegriff und Informationsträger bestimmt. Experimentelles Arbeiten mit Medientechnologien war der Avantgarde - den Medienkünstlern und Künstlerinnen - vorbehalten.

\section{Abstraktifizierung}

Die Computertechnologie hat durch Informatisierung eine Abstraktifizierung von Arbeits- und Anwendungsprozessen für den Nutzer mit sich gebracht. Informationen wurden formalisiert und abstraktifiziert, d. h. sie wurden zunehmend losgelöst von den konkreten, sinnlich-haptischen Weltbezügen, was kritisiert wurde «Der eigentliche Gegenstand, auf den sie sich beziehen, wird immer weniger konkret greifbar, wird im abstrakten Modell aufgehoben, wird subsumiert» (Kleemann

10 Beispielswiese führt der Medienpädagogische Forschungsverbund Südwest führt jährlich die JIMStudie durch, die seit 1998 jährlich den Medienumgang der 12-19-jährigen Jugendlichen untersucht. 
2000). Die Computerarbeit selbst wurde zum Gegenstand der Abstraktifizierung, mit Auswirkungen auf den Bildungsbereich. Die abstrakte Maschine und «Blackbox» Computer wurde im Informatikunterricht und in der technischen Bildung kaum thematisiert, bzw. transparent gemacht. Das Abstrakte wurde als nachvollziehbar vorausgesetzt, die Abstraktionsleistung von Schülerinnen und Schülern ebenso. Erst mit Einführung der Desktop-Metapher durch Macintosh wurde der Einsatz von Symbolen und Ikons realisiert. Die Abstraktifizierung von Rechenoperationen wurde im Kontext von Didaktik wenig problematisiert oder zu einem didaktisch relevanten Thema erhoben. Ganz im Gegenteil herrschte eine einseitige Sicht auf die Computertechnologie und Programmierung vor, die aus natur- und ingenieurwissenschaftlicher Perspektive geprägt war, wie es u. a. von Papert (1982) beschrieben wurde. Resnick formulierte später:

Today's blackbox instruments are highly effective in making measurements and collecting data - enabling even novices to perform advanced scientific experiments. But, at the same time, these black boxes are sopaque) (in that their inner workings are often hidden and thus poorly understood by their users) and they are bland in appearance (making it difficult for users to feel a sense of personal connection with scientific activity. (Resnick et al. 2000)

Dies war sein Ausgangspunkt für die Entwicklung von Crickets, programmierbare Bausteine, die Informationen von Sensoren auslesen, steuern und miteinander austauschen können. Auch von Seiten der Deutschen Gesellschaft für Informatik gab es Initiativen, das Abstrakte z. B. durch neuartige Schnittstellen, Programmierumgebungen und Interfaces begreifbarer zu machen, wie der Arbeitskreis «Begreifbare Interaktion» ${ }^{11}$ (vgl. Robben/Schelhowe 2012).

\section{Genderspezifische Medien- und Techniksozialisation}

Nach wie vor kann von einer genderspezifischen Medien- und Techniksozialisation ausgegangen werden, was sich an den Zahlen von Schülerinnen in technischen Leistungsfächern, Studentinnen in naturwissenschaftlich-technischen Disziplinen an Hochschulen, an der Berufswahl von Mädchen sowie an deren Selbstbildern («kein technischer Typ»), bzw. ihrer Einschätzung bezüglich ihrer technischen Kompetenzen und Fähigkeiten ablesen lässt, die sich oft auch mit den Vorstellungen der Eltern decken ${ }^{12}$. Demnach schätzt sich die Mehrzahl der weiblichen Teilnehmerinnen nicht als technikaffin ein, auch wenn sie in Hochschulseminaren oder im Unterricht motiviert Medienprojekte konzipieren, konstruieren, programmieren,

11 http://be-greifbar.de. Gründung des AK in der Gl 2007 (von Bruns, Schelhowe, Robben u. a).

12 Studie zur Berufsorientierung in fiktionalen Formaten «Berufsorientierung im Unterhaltungsformat» (Esch 2012). MINTiFF: Mathematik, Informatik, Natur- und Technikwissenschaften und Chancengleichheit im Fiction-Format: http://www.mintiff.de/content/0/58/59/63/page_3.html [30.7.2015] 
reflektieren und erfolgreich in Betrieb nehmen ${ }^{13}$. Andererseits liessen sich u.a. die Auseinandersetzung mit Programmierung im Kontext der Realisierung eigener Projektvorhaben mit ikonischen Programmierumgebungen (z. B. Amici, LEGO Mindstorms), Interesse am Unterrichtsgegenstand und damit verbunden das Einüben berufsunabhängiger Kompetenzen bei den Beteiligten beobachten (vgl. Reimann 2015). Es ist hinlänglich bekannt, dass Mädchen ihre eigenen Kompetenzen und Fähigkeiten bezüglich Technik und Computertechnologie eher als defizitär einschätzen. In Studien wurde (u. a. Buchen/Straub 2006) herausgearbeitet, dass geschlechtsspezifisches Verhalten weniger in (sozialisations- oder gar genetisch bedingten) Persönlichkeitsstrukturen wurzelt, sondern sich unter spezifischen Bedingungen und in konkreten Situationen auch im Hinblick auf das Technikverständnis jeweils neu hergestellt. Vielmehr wurzeln das vordergründige Technikinteresse, das fast ausschließlich bei (einigen) Jungen zu finden ist sowie die Distanzierung und der Rückzug auf einen zweckrationalen, gebrauchswertorientierten Zugang bei Mädchen eher in Selbstbildern, die Faszination und Leidenschaft (für Technik) nur für Jungen oder nur im homogenen Mädchenkontext zulassen bzw. im gemischtgeschlechtlichen Kontext für Mädchen hinderlich sind. (Schelhowe 2007, 131f.)

Auch neuere Initiativen, die vor allem dem Mangel an Fachkräften entgegengesetzt werden, zeigen wenig mädchenspezifische Förderungsschwerpunkte mit der Verbindung von Technik, Kunst und Gestaltung und lebensbedeutsamen, poetischen Zugangsweisen. Der Zugang von Mädchen zur Technik wird bei gestaltungsorientierten Ansätzen (wie z. B. IBP-GirlsLab, Reimann 2011) gerade nicht in einer grundsätzlichen Technikdistanz oder in Desinteresse vermutet, sondern in der Methodik und Vermittlungsarbeit, die nicht erst im Grundschulalter einsetzt. Auch das bundesweit angelegte und vom Fraunhofer Institut geförderte Projekt Roberta ${ }^{\circledR}$ nutzte It. eigener Aussage zwar «die Faszination von Robotern, um Schülerinnen Naturwissenschaften, Technik und Informatik spannend und praxisnah zu vermitteln» (zit. n. Roberta Website) ${ }^{14}$, führte aber nicht über traditionelle Vermittlungsansätze hinsichtlich einer Einbeziehung von künstlerischen Prozessen und Kontexten hinaus. Auch ein Projekt, das 2009 im Rahmen des bundesweiten Projekts Roberta ${ }^{\circledR}$ u. a. an der FH Kiel und an zwei Kieler Schulen durchgeführt wurde, setzte auf traditionelle Vermittlungsformen von robotischen Systemen: «Dabei lernen die jungen Menschen in einem ersten Schritt, die Roboter zusammenzubauen - alle bekommen die gleichen Bausätze [...]». Mädchenspezifische Kontexte und andere, z. B. ästhetisch-künstlerische Zugangsweisen werden dabei nicht thematisiert. Auf die Bedeutung der Kontextualisierung von robotischen Systemen und damit verbundene Möglichkeiten des Computers, Evokationen auszulösen bzw. mit ihm

13 Wie sich u. a. im GirlsLab zeigte (Reimann, 2015).

14 Vgl. Roberta- Lernen mit Robotern: Website: http://www.iais.fraunhofer.de/roberta.html [24.5.2010]. 
evokative Objekte zu erzeugen, wurde zunehmend im Kontext von Computern in Lernprozessen hingewiesen (Turkle 2008), fand aber selten Eingang.

\section{Ästhetisch-künstlerische Zugänge zur und informatisch-technische Lerninhal- te der Medienbildung}

Die identifizierten Grundprobleme stellen die Medienbildung vor Herausforderungen. Sie führten massgeblich zu der Entwicklung eines gestaltungsorientierten didaktischen Konzepts der Medienbildung mit dem Ziel ein ganzheitlich ausgeprägtes Technikverständnis bei Kindern und Jugendlichen zu entwickeln, indem an individuellen Fantasiewelten und lebensbedeutsamen Kontexten der Teilnehmerinnen und Teilnehmer anknüpft wurde. Zwecks Weiterführung und -verfolgung des Erkenntnisinteresses folgten dem Modellversuch ArtDeCom Forschungsprojekte und Initiativen, die den Ansatz weiterentwickelten, auf neue Technologien übertrug und mit weiteren pädagogischen Zielgruppen erprobte. In den Jahren 2004 bis 2006 wurde im Rahmen der Hochschulkooperation «Kids in Media and Motion (KiMM)» (Herczeg/Winkler 2004; IMIS der Universität zu Lübeck) das Forschungsprojekt «Kreative Medienkompetenz durch Hypermedia- und Mixed Reality-Systeme im Rahmen ästhetischer Forschungsprojektarbeit in Hochschule und Schule (MediaArtLab@School)» - im Hochschul- und Wissenschaftsprogramm als Folgeprojekt an der Universität Flensburg unter Einbeziehung der Kunstlehrer/ innenausbildung im Bereich der Grund-, Haupt- und Realschulen im Fach Kunst weitergeführt, an der Kunst- und Medienpädagogischen Projektschule in der UNESCO-Projektschule Flensburg praktisch mit Studierenden erprobt und evaluiert. Die Ergebnisse wurden regional in die Lehrer/innenaus- und -fortbildung einbezogen, auf Bundesebene sowie auf internationaler Bühne präsentiert und publiziert. (Reimann 2006, Reimann/Blohm 2005). KiMM-Initiative wurde weiterverfolgt und ein DFG-Schwerpunkt zum Lernen mit körper- und raumbezogenen Medien am IMIS der Universität zu Lübeck implementiert. ${ }^{15}$

Zur Kompensation der festgestellten Defizite in der Medienbildung wurde der gestaltungsorientierte Ansatz mit unterschiedlichen Zielgruppen didaktisch eingesetzt und evaluiert. Das BMBF-Forschungsprojekt «Künstlerisch-technische Medienbildung in Berufsvorbereitung und Berufsorientierung: Neue Ansätze zur Förderung digitaler Medienkompetenz von Jugendlichen (MediaArt@Edu: 2012 bis 2015)» reagierte auf die Forderung der BMBF-Expertenkommission (2010) nach Kompetenzbildung «in einer digital geprägten Kultur», in der explizit auf die Notwendigkeit einer «Medienbildung für die Persönlichkeitsentwicklung, für die

15 KiMM wurde im Rahmen des DFG-Sonderforschungsbereichs «Kinder- und jugendgerechte Vermittlungskonzepte von körper- und raumbezogenen interaktiven Computersystemen und Medien» am IMIS aus der Informatik heraus weitergeführt. 
gesellschaftliche Teilhabe und für die Entwicklung von Ausbildungs- und Erwerbsfähigkeit» hingewiesen wurde. Im Kern ging es der Kommission dabei auch um die Förderung eines Verständnisses der Wechselwirkungen zwischen virtueller und physikalischer Welt durch die Jugendlichen. Fragestellungen waren dabei, welche Kompetenzen junge Menschen in einer digital geprägten Kultur benötigen, wie sie sie erwerben können, welche Unterstützungsstrukturen dafür nötig sind bzw. geschaffen werden müssen und welche Anforderungen die Arbeitswelt vor dem Hintergrund zunehmender Medienkonvergenz (Wagner/Theunert 2006) stellt. Die BMBF-Expertenkommission wies in ihrem Papier explizit auf die neuen Möglichkeiten hin, die «durch Visualisierung, Interaktivität und Vernetzung entstehen, [und dass sie] neue Zugänge [zum Lernen] erleichtern und Motivationen wecken» können (2010, 11), was im Projekt aufgegriffen und realisiert wurde. Die kooperative Entwicklung von medialen Prototypen zu Bildungszwecken wurde dabei in den gestaltungsbasierten Ansatz eingebunden und mit ikonischen Formen des Programmierens realisiert, ohne dabei das ästhetische Handeln oder die künstlerischen Projektkontexte der präzisen Berechenbarkeit unterzuordnen. Darüber hinaus eröffnet Projekt- und Prototypenentwicklung eine Lernkultur, die Fehler als Ausgangspunkt für Lernprozesse nicht nur erlaubt, sondern einschliesst. Sie bedeutet in der pädagogischen Praxis die Entwicklung von Problemlösestrategien und unterstützt das Einüben kooperativer, überfachlicher Handlungen. (vgl. Reimann/Bekk 2015). Die Technik - selbst gestaltet, konstruiert, programmiert wird als ästhetische Erfahrung sinnlich-haptisch erlebbar. (Reimann et al. 2014, 216ff) Erprobt wurden u. a.

1. robotische Systeme, indem kreative Roboter-Konzepte entwickelt und mit Lego NXT realisiert wurden.

2. Games ON/OFF: Entwickeln von eigenen Spielumgebungen (VR) und Spielideen, die im realphysischen Raum gespielt werden, mit der Integration von StopMotion-Video, QR-Codes zur Vernetzung der Lernumgebung, «Makey makey» für die Neubelegung der Computertastatur mit physischen Objekten (Reimann/Bekk 2015)

3. Lichtobjekte: Entwerfen und gestalten mit verschiedenen Materialien und Farbeffekte programmieren. Die Software Chromoflex (Editor) unterstützte die Gestaltung von Farben und Effekte des Lichts, indem Farben und Effekte von den Lernenden programmiert und gemischt werden (siehe Reimann/Bekk 2014)

4. Smart Textilien (Wearables). Mittels Arduino-Technologie und einem schülergerechten Drag- and Drop-Interface (AMICl- Programmierumgebung) werden textile Objekte selbst geschneidert, mit einnähbaren Microcomputern ausgestattet (Arduino LilyPad), mit leitfähigem Stoff und Garn zu sich-verhaltenden Objekten arrangiert. (vgl. Reimann/Wüst 2014) 
5. Sound: Entwickeln von akusmatischen Klangcollagen (z. B. Aufnahme von Geräuschen aus der Umwelt, ihre digitale Bearbeitung und Erstellung einer eigenen Komposition (vgl. Reimann/Bekk 2016).

Dem Handeln mit Medien (Anwenden), der Visualisierung und Reflexion im Projektportfolio sowie der beruflichen Verwertbarkeit und der Lernprozessbegleitung von Jugendlichen in der ausserschulischen Berufsvorbereitung kamen im Projekt besondere Bedeutung zu. Sie wurden beim Lernen in Medienprojekten pädagogisch begleitet und wissenschaftlich beobachtet. Ziele des Projektes waren u. a. die Förderung von Technikverständnis und -interesse durch aktives Handeln mit Medien und visuelle Reflexion im Projektportfolio. Das Projektlernen stellte den Ausgangspunkt für das Einüben überfachlicher, berufsunabhängiger Fähigkeiten dar, die bei der Konzeption, Gestaltung, Konstruktion, Programmierung und In-Betriebnahme medialer Projekt-Prototypen angewendet wurden. Ziel war die Verbesserung der schwierigen Situation von Jugendlichen ohne Arbeit oder Ausbildung, an der Schnittstelle von berufs- und bildungsbiografischen Übergängen, ihrer aktiven Gestaltung durch die Betroffenen selbst und ihrer pädagogischen Begleitung.

Das Projekt hat erste Grundlagen der Erforschung von künstlerisch-technischen Ansätzen bei der Zielgruppe «Jugendliche in Berufsvorbereitungsund -orientierungsmaßnahmen> geleistet. Generell wurde der kunstpädagogisch basierte Ansatz des〈MediaArt@Edu〉-Projekts mit dem freien künstlerischen Bearbeiten offener Aufgabenstellungen von den beteiligten Jugendlichen außerschulischer Berufsvorbereitung sehr positiv angenommen [...]. Generell stellten die eingesetzten Medien und Technologien einen Motivator für die Jugendlichen dar, der ihr Interesse weckte und durch den spielerischen Ansatz auch für weniger technikerfahrende und technikaffine Jugendliche reizvoll war. Trotz der gemeisterten Tätigkeiten bei der kooperativen Konzeption, Entwicklung, Gestaltung, Konstruktion und Inbetriebnahme der eigenen Projekte, hatten die Jugendlichen kaum ein Bewusstsein über die eigenen Fähigkeiten, ihre Bedeutung für die Zukunft und ihre berufliche Verwertbarkeit, so dass eine angeleitete Reflexion essentiell erforderlich war, um Teilnehmenden zu ermöglichen, eigene Ressourcen und Stärken nicht nur im Projektkontext zu erleben und anwenden zu lassen, sondern ihnen diese auch explizit als Könnerschaft vor Augen zu führen. (Reimann/Bekk 2016, 36) 


\section{Programmierkompetenz als Thema für die Lehrerbildung}

Nicht nur die Technologien, sondern auch berufliche Tätigkeiten und Qualifikationsprofile ändern sich im Zuge des technischen Wandels und der Entwicklung zur sogenannten «Industrie 4.0». Die Fachkräfte von morgen müssen in der Lage sein, aktuelle Technologien einzusetzen, zu bedienen und Störungen auszuräumen. Auszubildende müssen z.B. mit 3D-Druckern, Assistenzsystemen oder Drohnen umgehen können. Dies erfordert eine entsprechende Anpassung der «Lehrerbildung 4.0». Auf der Seite der Lehrkräfte ist die praktische Medienkompetenz noch recht unterschiedlich entwickelt, häufig werden digitale Technologien im Unterricht noch negativ konnotiert. Dass die Förderung der Programmierfähigkeit als Gegenstand der Lehrerbildung inzwischen auch auf europäischer Bühne verfolgt wird, ist vor allem mit der Konkurrenzfähigkeit des Standorts begründet und folgt der Beobachtung von Initiativen einzelner, nicht nur europäischer Länder. In Estland lernen Schülerinnen und Schüler bereits von der ersten Klasse an das Programmieren und die Programmierung von Computern wurde bereits für alle Altersgruppen in den Lehrplänen verankert. Dänemark hat dies teilweise ebenso eingebracht. Andere europäische Länder sind im Begriff, es einzuführen (wie Großbritannien im Jahr 2014) und andere ziehen es in Betracht (z. B. Finnland und Irland). In vielen Ländern, die Programmierung zwar nicht als Teil des Pflichtlehrplans verankern, gibt es einzelne Lehrkräfte, die versuchen, das Thema Programmierung in den Unterricht einzuführen. Was unvermeidlich zu sein scheint, ist, dass sich alle EUMitgliedstaaten in diese Richtung bewegen, um den Anforderungen des europäischen Arbeitsmarktes gerecht zu werden. Eine grosse Herausforderung stellt dabei der Mangel an qualifizierten Lehrkräften dar. Mathematik- und InformatikAbsolventen wählen in der Regel lukrativere Berufe, Absolventen der Geistes- und Sozialwissenschaften, die Programmieren unterrichten, benötigen viel Unterstützung, ebenso wie die Lehrkräfte an Grundschulen (vgl. Vermeersch/Hughes 2015). Auf EU-Ebene wird "Coding» im Rahmen des ökonomischen Standorts und der allgemeinen Konkurrenzfähigkeit u. a. mit der «Code week 2014» der Europäischen Kommission grossflächig als Bildungsziel lanciert, 2015 weitergeführt und im Kontext von Arbeit und Spiel wahrgenommen:

Why learn to code? In a world where we're surrounded by technology, and where so many of our interactions we have are with computers, learning to code helps us understand how these services work. What's more, learning to code gives us a powerful way to explore our ideas and make things, both for work and play. (zit. n. Website www.codeweek.eu).

Daran anknüpfend wurde "TACCLE3 coding- Teachers' Aids on Creating Content for Learning Environments» ${ }^{16}$ aufgelegt, eine europäische Projektkonstellation, die die Integration von Programmierkompetenz als Unterrichtsgegenstand

16 Das Forschungsprojekt wird im Erasmus+ Programm von 2015-2017 gefördert. www.taccle3.eu. 
in die Lehrerfortbildung integriert. Es reagiert auf den festgestellten Mangel an informatisch bzw. informationstechnologisch ausgebildeten Grundschullehrkräften. Das Projekt zielt auf die Erstellung grundlegender Didaktischer Materialien zwecks Förderung der Programmierfähigkeit durch geeigneten Unterricht. Weitere Ziele sind die Unterstützung von (vornehmlich informatikfernen) Lehrkräften, Programmierfähigkeit als informatisches Denken und Handeln anhand von geeigneten Lernmaterialien zu unterrichten und diese systematisch an Grundschulen (K4-14-Klassen) einzusetzen, um die Schülerinnen und Schüler zu ermöglichen, Programmierkompetenz zu erwerben, Technikverständnis auszubilden und die erworbenen Fähigkeiten später einsetzen zu können. Im Rahmen des ErasmusProjekts wurden in den beteiligten Ländern Belgien, Grossbritannien, Estland, Finnland und Deutschland Medien, Materialien, Ressourcen und Werkzeuge recherchiert, aufbereitet, online für Lehrkräfte bereitgehalten und in Form von einer Lehrerfortbildungswoche an die Zielgruppe vermittelt (Vermeersch/Hughes 2015; García-Peñalvo 2016). In Deutschland, wo Programmierkompetenz noch nicht in Lehrpläne von Grundschulen integriert wurde, wurden ästhetisch-spielerische Zugänge für da Unterrichten von Programmierkompetenz sowie Unterrichtsaktivitäten für jüngere Schülerinnen und Schüler entwickelt (vgl. Reimann/Maday 2016). Dabei wurden u. a. LilyPad-Arduino-Technologie und die sinnlich-haptischen Gestaltungsmöglichkeiten interaktiver Textilien didaktisch eingesetzt. «TACCLE3 coding» zielt darauf ab, die Qualifikationsbasis und Fachkompetenz der Lehrenden an Schulen zu verbessern, indem ein Online-Support-Angebot für Lehrende mit Ideen, Unterrichtsaktivitäten, Materialien und Ressourcen bereitgehalten werden, die das Lehren von Programmierfähigkeit unterstützen und Lehrkräften die Produktion eigener Unterrichtsstunden und Module in den beteiligten Ländern und Regionen erleichtern (vgl. Vermeersch/Hughes 2015).

\section{Fazit und Ausblick}

Die reale Lebenswelt wird mit all ihren Entäusserungen im virtuellen Raum abgebildet und mit ihm verknüpft: Der Ort, wo ich bin, das, was ich tue, genauso wie, mit wem ich kommuniziere und was ich kaufe. Datenspuren im Virtuellen, die immer genauer erfassen, was wir tun, werden gespeichert, vernetzt und an Dritte gesendet. Gleichzeitig erhalten die Subjekte in der digitalisierten Welt immer passgenauere Vorschläge und Angebote von Assistenzsystemen aus dem virtuellen Raum. Das Virtuelle beeinflusst das Reale in immer stärkerem Ausmass, es ist aber keine «neutrale Welt», sondern wird von Konzernen und ihren Geschäftsinteressen vorangetrieben. Trotz dieser fortschreitenden Digitalisierung der Welt und der damit verbundenen Bedeutung der zugrundeliegenden algorithmischen Strukturen - und trotz der positiven Evaluation geeigneter Lehr-Lernkonzepte und 
neuer Zugänge zur Integration von informatischen Lerninhalten, finden sich in der medienpädagogischen Diskussion in Praxis und Forschung ausserhalb spezifischer Projekte noch wenig konkrete Auseinandersetzungen mit dem Bereich der «Programmierfähigkeit». Im Gegenteil: Längst als überholt betrachtete Vermittlungsformen - z. B. ein stark instrumentelles Computerverständnis, die werkzeugorientierte, auf Anwenderkompetenzen ausgerichtete Thematisierung, Rezeption und Verwendung Digitaler Medien - die zwar inzwischen überholt anmuten mögen, halten sich trotz aller disziplinübergreifenden und -verbindenden Bemühungen als Realität in den heutigen Schulen, Hochschulen und in ausserschulischen Kontexten. Die vorgestellten Ansätze und Projektergebnisse, die von den unterschiedlichen pädagogischen Zielgruppen gut angenommen wurden, stehen für die Medienpädagogik an Schule, Hochschule und in ausserschulischen Settings bereit. Sie können aufgenommen und eingesetzt werden, um eine nachhaltige und systematische Integration informatischer und medienpädagogischer Inhalte und ihre curriculare Implementierung, z. B. im Rahmen einer medienpädagogischen Grundbildung an Hochschulen und Schulen zu realisieren.

\section{Literatur}

Alemann, Ulrich von, und Heribert Schatz. 1986. Mensch und Technik: Grundlagen und Perspektiven einer sozialverträglichen Technikgestaltung. Bd. Band 1. Sozialverträgliche Technikgestaltung. Opladen: Westdeutscher Verlag.

Amelunxen, Hubertus, und Michael Herczeg. 2001. «Die Epistemologie der Medienkunst». In Mensch \& Computer 2001, hrsg. v. Horst Oberquelle, Reinhard Oppermann, und Jürgen Krause, 55:397-98. Wiesbaden: Vieweg+Teubner Verlag. doi:10.1007/978-3-32280108-1_46.

Anderson, Chris. 2013. Makers: Das Internet der Dinge: die nächste industrielle Revolution. Übersetzt von Sigrid Schmid. München: Carl Hanser Verlag GmbH \& Co. KG. doi:10.3139/9783446436374.

BMBF - Bundesministerium für Bildung und Forschung, Hrsg. 2010. «Kompetenzen in einer digital geprägten Kultur. Medienbildung für die Persönlichkeitsentwicklung, für die gesellschaftliche Teilhabe und für die Entwicklung von Ausbildungs- und Erwerbsfähigkeit». http://www.dlr.de/pt/Portaldata/45/Resources/a_dokumente/bildungsforschung/ Medienbildung_Broschuere_2010.pdf.

Buchen, Sylvia, und Ingo Straub. 2006. «Die Bedeutung des Hacker-Topos für Hauptschüler in der Adoleszenz». In Gender medienkompetent, hrsg. v. Annette Treibel, Maja S. Maier, Sven Kommer, und Manuela Welzel, 93-110. Wiesbaden: VS Verlag für Sozialwissenschaften.

Buechley, Leah, Mike Eisenberg, und Nwanua Elumeze. 2007. «Towards a Curriculum for Electronic Textiles in the High School Classroom». ACM SIGCSE Bulletin 39 (3): 28. doi:10.1145/1269900.1268795.

Coy, Wolfgang. 2008. «Kulturen - nicht betreten? Anmerkungen zur «Kulturtechnik Informatik»». Informatik-Spektrum 31 (1): 30-34. doi:10.1007/s00287-007-0207-z. 
Dahlmann, Frank. 2014. «Hacker School: Eine Idee macht Schule. Aus der Not entstand eine beispielhafte Privatinitiative: die Hacker School». brand eins 9 (Schwerpunkt Arbeit). https://www.brandeins.de/archiv/2014/arbeit/hacker-school-david-cummins-andreasollmann-timm-peters-eine-idee-macht-schule/.

Esch, Marion. 2012. «Berufsorientierung im Unterhaltungsformat». http://www.mintiff.de/ content/0/58/59/63/page_3.html.

Garcia-Penalvo, Francisco J. 2016. «A brief introduction to TACCLE 3 - coding European project». 1-4. IEEE. doi:10.1109/SIIE.2016.7751876.

Herczeg, Michael, und Thomas Winkler. 2004. «Kids in media and motion. Lernen mit den Medien von morgen». http://www.kimm.uni-luebeck.de/.

Kafai, Yasmin B., und Quinn Burke. 2014. Connected Code: Why Children Need to Learn Programming. The John D. and Catherine T. MacArthur Foundation Series on Digital Media and Learning. Cambridge, Mass: The MIT Press.

Kerres, Michael. 2012. Mediendidaktik: Konzeption und Entwicklung mediengestützter Lernangebote. 3. Aufl. München: Oldenbourg Wissenschaftsverlag. doi:10.1524/9783486716924.

Kleemann, Frank. 2000. «Informatisierung der Arbeit: Folgen für Arbeitsverhältnisse und subjektive Leistungen der Arbeitenden».Kommunikation@gesellschaft 1 (B3): 1-11.

Kreye, Andrian. 2014. "Neue Weltsprache». sueddeutsche.de, Juli 22, Abschn. digital. http://www.sueddeutsche.de/digital/bedeutung-von-algorithmen-neue-weltsprache-1.2051528.

Manovich, Lev. 2013. Software takes command: extending the language of new media. International texts in critical media aesthetics. New York; London: Bloomsbury. http://softwarestudies.com/softbook/manovich_softbook_11_20_2008.pdf.

Niesyto, Horst, und Heinz Moser. 2009. «Medienpädagogisches Manifest - Keine Bildung ohne Medien!» Hrsg. v. Kommission Medienpädagogik in der Deutschen Gesellschaft für Erziehungswissenschaft, Fachgruppe Medienpädagogik in der Deutschen Gesellschaft für Publizistik- und Kommunikationswissenschaft, Vorstand der Gesellschaft für Medienpädagogik und Kommunikationskultur (GMK), Vorstand des JFF - Jugend, Film, Fernsehen e.V., und Hans-Bredow-Institut für Medienforschung. http://www.keine-bildung-ohne-medien.de/pages/medienpaed-manifest/.

Papert, Seymour. 1982. Mindstorms: Kinder, Computer und Neues Lernen. Basel u.a: Birkhäuser.

Pazzini, Karl-Josef. 1999. Kulturelle Bildung im Medienzeitalter: Gutachten zum Programm. Herausgegeben von Bund-Länder-Kommission für Bildungsplanung und Forschungsförderung. Materialien zur Bildungsplanung und zur Forschungsförderung 77. Bonn: BLK, Geschäftsstelle. http://www.blk-bonn.de/papers/heft77.pdf.

Przybylla, Mareen, und Ralf Romeike. 2013. «Physical Computing im Informatikunterricht». In Informatik erweitert Horizonte, INFOS 2013, 15. Gl-Fachtagung Informatik und Schule, 26.-28. September 2013, Kiel, Germany, hrsg. v. Norbert Breier, Peer Stechert, und Thomas Wilke, P-219:137-146. LNI. GI. http://subs.emis.de/LNI/Proceedings/Proceedings219/article7.html.

Reimann, Daniela. 2006. Ästhetisch-informatische Medienbildung mit Kindern und Jugendlichen: Grundlagen, Szenarien und Empfehlungen für Gestaltungsprozesse in Mixed Reality-Lernräumen. Bd. 23. Artificium. Oberhausen: Athena. 
Reimann, Daniela. 2011. «Shaping Interactive Media with the Sewing Machine: Smart Textile as an Artistic Context to Engage Girls in Technology and Engineering Education». International Journal of Art, Culture and Design Technologies 1 (1): 12-21. doi:10.4018/ ijacdt.2011010102.

Reimann, Daniela. 2015. «Smart Textile as a Creative Environment to Engage Girls in Technology». In Analyzing Art, Culture, and Design in the Digital Age, hrsg. v. Gianluca Mura, 205-17. Hershey, PA: IGI Global. doi:10.4018/978-1-4666-8679-3.

Reimann, Daniela, und Simone Bekk. 2014. «Künstlerisch geleitete Medienbildung mit Portfolios: Potenziale für Jugendliche in berufsvorbereitenden Bildungsmaßnahmen. Herausforderungen beim Übergang Schule - Beruf und das Konzept der berufsbiografischen Gestaltungskompetenz». hrsg. v. Theo Hug, Petra Missomelius, und Wolfgang Sützl. medienimpulse. Beiträge zur Medienpädagogik 2 (Medienpädagogische Potenziale digitaler Medienkunst). http://www.medienimpulse.at/articles/view/659.

Reimann, Daniela, und Simone Bekk. 2015. "Game Design with Portfolios and Creative Skills». In Creativity in the Digital Age, hrsg. v. Nelson Zagalo und Pedro Branco, 245-61. London: Springer London. doi:10.1007/978-1-4471-6681-8_13.

Reimann, Daniela, und Simone Bekk. 2016. «Künstlerisch-technische Medienbildung im BMBF-Forschungsprojekt 〈MediaArt@Edu〉: Ansatz, Ziele und Ergebnisse». In Gestaltungsorientierte Aktivierung von Lernenden: Übergänge in Schule - Ausbildung - Beruf, hrsg. v. Daniela Reimann, Simone Bekk, und Martin Fischer, 15-44. Norderstedt: BoD Books on Demand.

Reimann, Daniela, und Manfred Blohm. 2005. «Game on- Mixed Reality-Game Development in Media Art Education at School and University». In Proceedings of the Conference on Complex Research on Creativity and Implementation of its Results in Educational Practice. Riga: Teacher Training and Management School.

Reimann, Daniela, und Christiane Maday. 2016. «Smart Textile Objects and Conductible Ink as a Context for Arts Based Teaching and Learning of Computational Thinking at Primary School». ACM Press. 31-35. doi:10.1145/3012430.3012493.

Reimann, Daniela, Thomas Winkler, Michael Herczeg, und Ingrid Höpel. 2003. «Exploring the Computer as a Shapeable Medium by Designing Artefacts for Mixed Reality-Environments in Interdisciplinary Education Processes». In Proceedings of EdMedia: World Conference on Educational Media and Technology 2003, hrsg. v. David Lassner und Carmel McNaught, 915-922. Honolulu, Hawaii, USA: Association for the Advancement of Computing in Education (AACE). https://www.learntechlib.org/p/13911.

Reimann, Daniela, Thomas Winkler, Michael Herczeg, und Ingrid Höpel. 2004. «Medien als Schnittstelle zwischen Kunst und Informatik im Kontext künstlerischer Konzepte und erweiterter Kunst- und mediendidaktischer Vermittlungsfelder». In Künstlerische Bildung nach Pisa. Beiträge zum Internationalen Symposium «Mapping Blind Spaces - Neue Wege zwischen Kunst und Bildung), hrsg. v. Joachim Kettel, und Internationale Gesellschaft der Bildenden Künste (IGBK), Landesakademie Schloss Rotenfels. Museum für Neue Kunst im ZKM | Zentrum für Kunst und Medientechnologie Karlsruhe und Landesakademie Schloss Rotenfels 08.-10.10.2003, Oberhausen.

Reimann, Daniela, und Andrea Wüst. 2014. «Konzepte für eine ästhetisch-künstlerisch geleitete Berufsorientierung mit interaktiven Textilien». BDK-Mitteilungen. Fachzeitschrift des BDK - Fachverband für Kunstpädagogik 3: 14-17. 
Reimann, Daniela, Andrea Wüst, und Miriam Burkhart. 2014. «Digitale Medien als ästhetische Erfahrungsräume für Jugendliche in berufsvorbereitenden Bildungsmaßnahmen Portfoliodesign und -mentoring entlang der Gestaltungsprozesse». In Jahrbuch Medienpädagogik 11. Diskursive und produktive Praktiken in der digitalen Kultur, hrsg. v. Rudolf Kammerl, Alexander Unger, Petra Grell, und Theo Hug, 11:209-30. Jahrbuch Medienpädagogik. Wiesbaden: Springer Fachmedien Wiesbaden. doi:10.1007/978-3658-06462-4_12.

Resnick, Mitchel, Robbie Berg, und Michael Eisenberg. 2000. «Beyond Black Boxes: Bringing Transparency and Aesthetics Back to Scientific Investigation». Journal of the Learning Sciences 9 (1): 7-30. doi:10.1207/s15327809jls0901_3.

Richard, Jörg, und Willi Bruns. 2004. «Soll Ästhetische Erziehung ein `SmartSpiel« werden?» Ästhetik und Kommunikation 125: 69-76.

Robben, Bernard, und Heidi Schelhowe, Hrsg. 2012. Be-greifbare Interaktionen: Der allgegenwärtige Computer: Touchscreens, Wearables, Tangibles und Ubiquitous Computing. Kultur- und Medientheorie. Bielefeld: transcript Verlag. doi:10.14361/ transcript.9783839420058.

Roßmann, Robert. 2015. «Bildung am Bildschirm». sueddeutsche.de, Abschn. bildung. http://www.sueddeutsche.de/bildung/schule-bildung-am-bildschirm-1.2406259.

Schelhowe, Heidi. 2007. Technologie, Imagination und Lernen: Grundlagen für Bildungsprozesse mit digitalen Medien. Münster: Waxmann.

Schön, Sandra, Martin Ebner, und Kristin Narr. 2016. Making-Aktivitäten mit Kindern und Jugendlichen: Handbuch zum kreativen digitalen Gestalten. Norderstedt: Books on Demand.

Smith, David Canfield. 1975. «PYGMALION: A Creative Programming Environment». Dissertation, Stanford, California, USA: Computer Science Department, Stanford Artificial Intelligence Labratory. Stanford University.

Turkle, Sherry. 1984. Die Wunschmaschine: vom Entstehen der Computerkultur. Übersetzt von Nikolaus Hansen. 1. Aufl. Reinbek bei Hamburg: Rowohlt.

Turkle, Sherry, Hrsg. 2008. Falling for Science: Objects in Mind. Cambridge, Mass: MIT Press. Vermeersch, Jens, Jenny Hughes. 2015. «Teachers' Aids on Creating Content for Learning Environments (TACCLE) 3 Coding. Proposal to the European Commission, Erasmus+ program, key action: Cooperation for innovation and the exchange of good practices». Brussels: GO!, Submission to the European Commission Erasmus+ KA1 program.

Wagner, Ulrike, Helga Theunert, und Bayerische Landeszentrale für Neue Medien, Hrsg. 2006. Neue Wege durch die konvergente Medienwelt: Studie im Auftrag der Bayerischen Landeszentrale für neue Medien (BLM). BLM-Schriftenreihe 85. München: Fischer. 